\title{
Observation of Faraday rotation from a single confined spin
}

\author{
METE ATATÜRE* ${ }^{\star \dagger}$, JAN DREISER*, ANTONIO BADOLATO AND ATAC IMAMOGLU ${ }^{\dagger}$ \\ Institute of Quantum Electronics, ETH Zurich, CH-8093 Zurich, Switzerland \\ *These authors contributed equally to this work \\ †e-mail: atature@phys.ethz.ch; imamoglu@phys.ethz.ch
}

Published online: 28 January 2007; doi:10.1038/nphys521

The ability to read out the state of a single confined spin lies at the heart of solid-state quantum-information processing ${ }^{1}$. Although spin measurements using Faraday rotation of light polarization have been implemented in semiconductor spin ensembles $^{2-4}$, single-spin read-out has only been achieved using transport measurements ${ }^{5,6}$. Here, we demonstrate an all-optical dispersive measurement of the time-averaged spin state of a single electron in a quantum dot. We obtain information on the spin state through conditional Faraday rotation of a spectrally detuned laser, induced by the polarization- and spin-selective trion (charged quantum dot) transitions. To assess the sensitivity of the technique, we use an independent resonant laser for spinstate preparation ${ }^{7}$. We infer that there are $\sim 10$ spin-flip Raman scattering events (that is, back-action) within our measurement timescale. Straightforward improvements such as incorporating solid-immersion lenses ${ }^{8,9}$ and higher efficiency detectors should allow for back-action-evading spin measurements, without the need for a cavity.

Absorption and dispersion coexist in an optical field's response to a spectrally detuned optical transition. Although these responses are of comparable strength for small detunings, dispersive response dominates over the absorptive part as the detuning is increased. Measurement of the dispersive response provides information about the ground (spin) state, if the transition obeys spin-selective optical selection rules, as is the case for a quantum dot (QD) confining a single excess electron ${ }^{7,10}$. Here, we demonstrate measurement of a QD spin by detecting this dispersive response through Faraday rotation (FR) of a far-detuned linearly polarized laser.

Theoretical proposals on the basis of FR from a microcavityembedded QD have suggested that quantum non-demolition ${ }^{11}$ measurement of a single spin could be implemented ${ }^{12,13}$. Remarkably, our observations suggest that the back-action-evading spin measurement in the basis defined by the Pauli operator $\sigma_{z}$ (the strong confinement axis) could be realized even in the absence of an optical cavity enhancing the FR. We estimate that the QD scatters a photon every $2 \mu \mathrm{s}$; the role of these events is to leak state information into the field reservoir, thus, inducing back-action on the $\sigma_{x}$ observable and collapsing the state into a $\sigma_{z}$ eigenstate. The spin-flip Raman scattering events, which lead to back-action on the measured observable $\left(\sigma_{z}\right)$, occur once every 2 to $20 \mathrm{~ms}$. Although it is impossible to avoid Rayleigh scattering within a measurement time yielding a signal-to-noise ratio exceeding unity, spin-flip scattering can be negligible if the ratio of the peak absorption cross-section and the laser area $\left(\sigma_{\mathrm{QD}} / A_{\mathrm{L}}\right)$ considerably exceeds the ratio of the Rayleigh and spin-flip Raman scattering rates (that is, the branching ratio into the spin-flip scattering channel $)^{7}$. We have determined that the branching ratio in self-assembled QDs is between $10^{-3}$ and $10^{-4}$ and is primarily due to hyperfine-induced state mixing.

Spin measurements could also be carried out using resonance fluorescence or absorption experiments. However, owing to background Rayleigh scattering and limited collection efficiency, the timescales needed to measure the spin state via the resonance fluorescence technique exceed the spin-flip timescales. Resonant absorption measurements are likewise limited by electrical noise that dominates over the shot noise for laser intensities that do not saturate the QD transitions. FR, however, allows for shot-noiselimited spin measurements with the highest signal-to-noise ratio among these techniques, by a proper choice of laser intensity and detuning. Furthermore, for QDs embedded in cavities, FR provides the only measurement scheme that can avoid light scattering completely; this is crucial in measurement-induced-entanglement schemes or in systems with a considerable branching ratio into the spin-flip scattering channel.

A single electron confined in a QD presents a four-level system in the trion representation, as illustrated in Fig. 1a. The ground state consists of an electron in the $|\downarrow\rangle(|\uparrow\rangle)$ state. The excited state $|\uparrow \downarrow, \boldsymbol{\nabla}\rangle(|\uparrow \downarrow, \mathbf{\Delta}\rangle)$ corresponds to the QD with two electrons forming a singlet and a hole with angular momentum projection $J_{z}=-3 / 2(3 / 2)$ along the growth direction. The trion transition, $|\uparrow \downarrow, \boldsymbol{\nabla}\rangle-|\downarrow\rangle(|\uparrow \downarrow, \mathbf{\Delta}\rangle-|\uparrow\rangle)$, is allowed only for $\left[\sigma^{(-)}\right]\left(\left[\sigma^{(+)}\right]\right)$ circular polarization as determined by the optical selection rules. If a linearly polarized laser-for example, $\left[\pi^{(+)}\right]$-feels this transition, the $\left[\sigma^{(-)}\right]\left(\left[\sigma^{(+)}\right]\right)$polarization component acquires a phase shift rotating the laser's polarization by an angle $\theta(-\theta)$ in the linear basis. Owing to Pauli blockade ${ }^{14}$, only one of these transitions is available at any given time and the laser polarization is rotated in the positive or negative angular direction depending on the electron's spin state. In our experiments, we exploit precisely this spin-state-dependence of the laser polarization.

Figure $1 \mathrm{~b}$ shows our detection scheme. A polarized laser propagates through a gated heterostructure incorporating QDs. A polarizing beam splitter distributes the transmitted light into two linear polarizations in the rectilinear basis of $(\mathrm{X}, \mathrm{Y})$ and directs each arm to a photodiode. Along with each detector's output $\left(T_{x}\right.$ and $T_{y}$ ), such a configuration allows us to measure their sum and difference simultaneously. The sum $\left(T_{\text {sum }}\right)$ normalized to the total incident laser intensity indicates a change of photon number in the total light, yielding the absorptive response, whereas the 

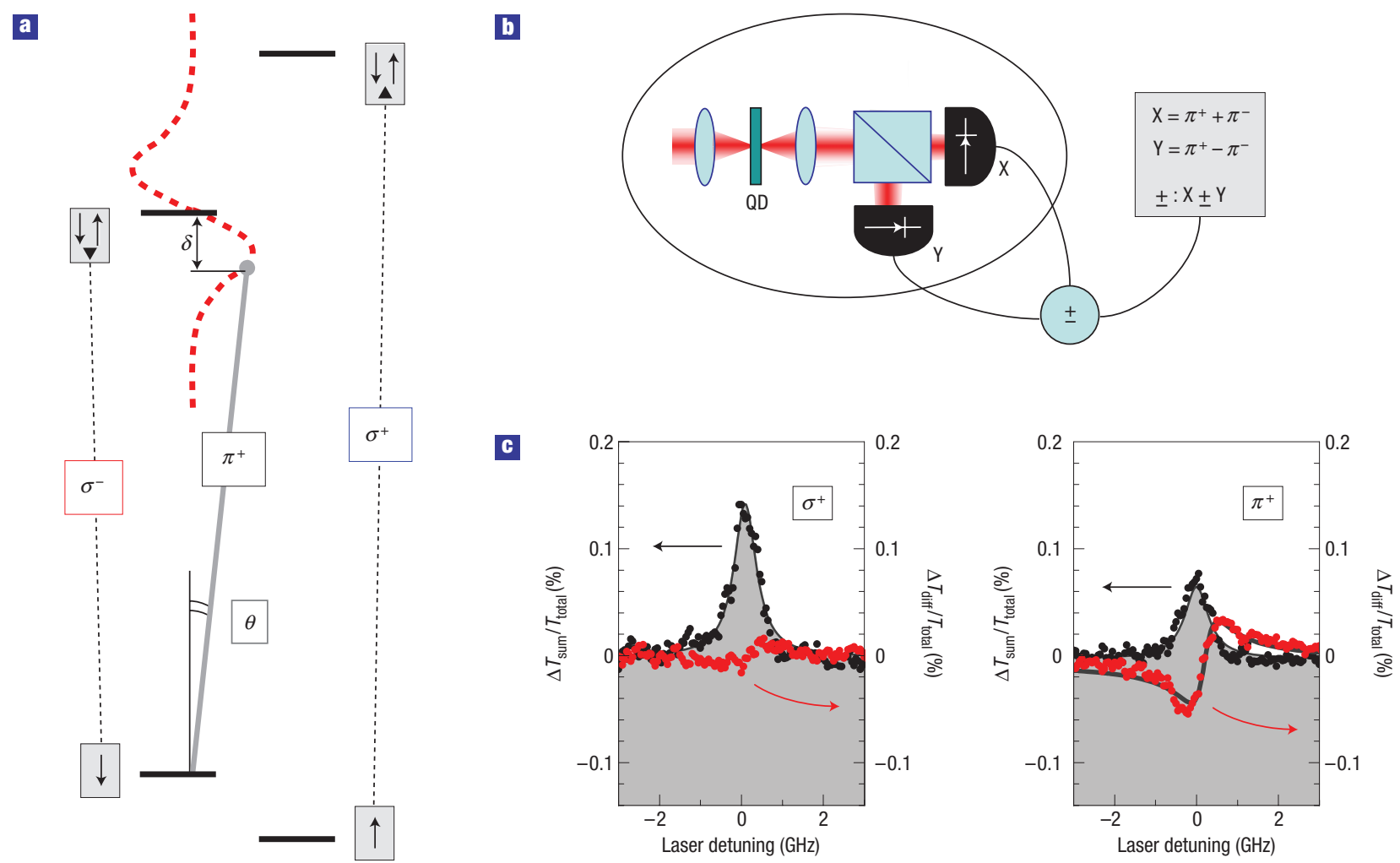

Figure 1 QD transitions and their response to an off-resonant laser. a, The ground and excited states of a single-electron-charged QD can be represented by a four-level scheme, where each ground state is linked to an excited state through a circularly polarized laser owing to the optical selection rules. A linearly polarized optical field detuned by $\delta$ from the resonance experiences a polarization rotation due to the Faraday effect. $\mathbf{b}$, In the experimental apparatus, a laser beam is impingent on the sample (kept at $4.2 \mathrm{~K}$ ) with a single QD in the focused laser area. On transmission, the laser is distributed by a polarizing beam splitter to two detectors. c, The left (right) figure shows differential transmission data from a single-electron-charged $Q D$ at $1 \mathrm{~T}$ external magnetic field obtained using a circularly (linearly) polarized laser. The black circles represent the sum of two detector signals, which is proportional to the absorptive response, whereas the red circles represent the difference of the two detector signals, proportional to the dispersive response. The probe-laser power is $20 \mathrm{nW}$, corresponding to a Rabi frequency $\Omega_{\mathrm{L}}^{2} \sim \gamma^{2} / 2$ on resonance, and the relative value of absorption is $0.15 \%$ for circular polarized excitation at this power level.

(normalized) difference of the two signals $\left(T_{\text {diff }}\right)$ is linked to the polarization rotation arising from the dispersive response.

In the absence of an external magnetic field, the trion transition has a twofold spin degeneracy. Therefore, although we fully observe the absorptive response in the summed detector signals, the dispersive response is exactly cancelled owing to the fast spinflips induced by the hyperfine interaction ${ }^{15}$. Under an external magnetic field however, the Zeeman splitting $\left(E_{\text {Zeeman }}\right)$ lifts the spectral degeneracy of the two spin-selective trionic transitions (as in Fig. 1a). Consequently, the dispersive response is expected to appear along with the absorptive part in the spectral vicinity ( $\left.\Delta \ll E_{\text {Zeeman }}\right)$ of one of the Zeeman-split transitions in the form

$$
T_{\text {diff }}=\theta[\mathrm{rad}]=\alpha_{0} \Delta(\gamma / 2) /\left[\Delta^{2}+(\gamma / 2)^{2}\right]
$$

where $\alpha_{0}$ is proportional to the ratio $\sigma_{\mathrm{QD}} / A_{\mathrm{L}}$ and incorporates heterostructure-induced mode mismatch, $\gamma$ is the total linewidth measured in absorption measurements, $\Delta$ is the spectral detuning of the probe laser and $\theta$ is the FR angle.

Figure 1c shows how the sum (black circles) and difference (red circles) signals from one of the Zeeman-split transitions behave as a function of laser detuning for two different polarizations, when an external magnetic field of $1 \mathrm{~T}$ is applied along the strong confinement axis of the QD. The transitions are split by $26 \mathrm{GHz}$, which is $\sim 60$ times larger than the total transition linewidth $\gamma$, and the laser's response in the near vicinity of the $\left[\sigma^{(-)}\right]$-polarized transition linked to the spin-down electronic state is shown. When the laser is also circularly polarized (left plot), it acquires an overall phase that cannot be detected leading to a purely absorptive signal (black circles). The same scan with a linearly polarized laser (right plot) shows the dispersive response (red circles) alongside the absorptive response (black circles): as the acquired phase is now relative, it leads to a linear polarization rotation. The solid grey lines are fits using equation (1) for the dispersive QD response. Both curves in the $\left[\pi^{+}\right]$case have $\alpha_{0}=0.14 \%, \Omega_{\mathrm{L}}^{2}=\gamma^{2} / 2$ and $\gamma=2 \pi \times 466 \mathrm{MHz}$, as obtained from line-broadening in saturation spectroscopy, where full-width at half-maximum $=2 \times\left\{(\gamma / 2)^{2}+\Omega_{\mathrm{L}}^{2} / 2\right\}^{1 / 2}$. At such small detuning with respect to Zeeman splitting, the laser experiences FR primarily due to one Zeeman transition nearby. The data presented here are obtained in the cotunnelling regime to avoid spin pumping ${ }^{7}$. We note here that similar dispersive response signatures of single QDs have recently been reported ${ }^{16}$. In contrast to the work presented here, the dispersive signals observed there ${ }^{16}$ are polarization and spin independent and arise from an interference effect that depends on the QD position with respect to the laser focal spot in a planar Fabry-Perot cavity. 


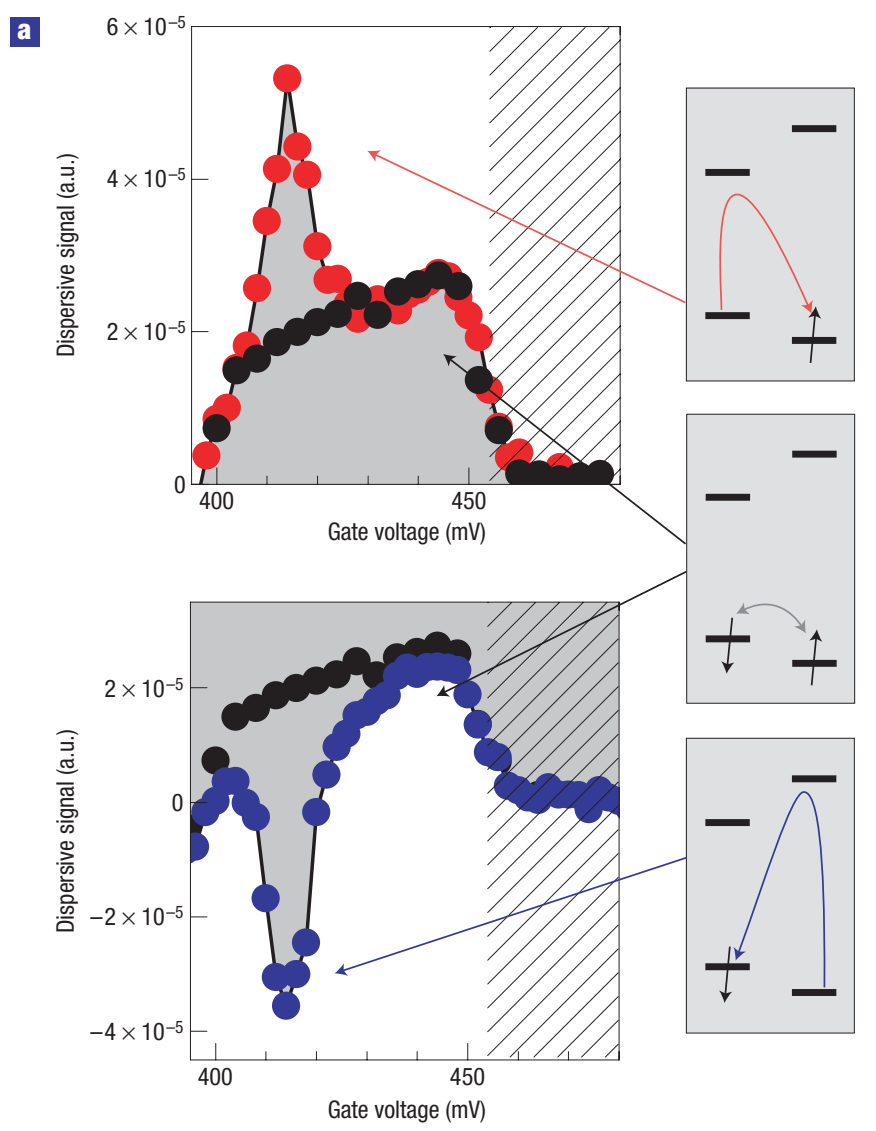

b

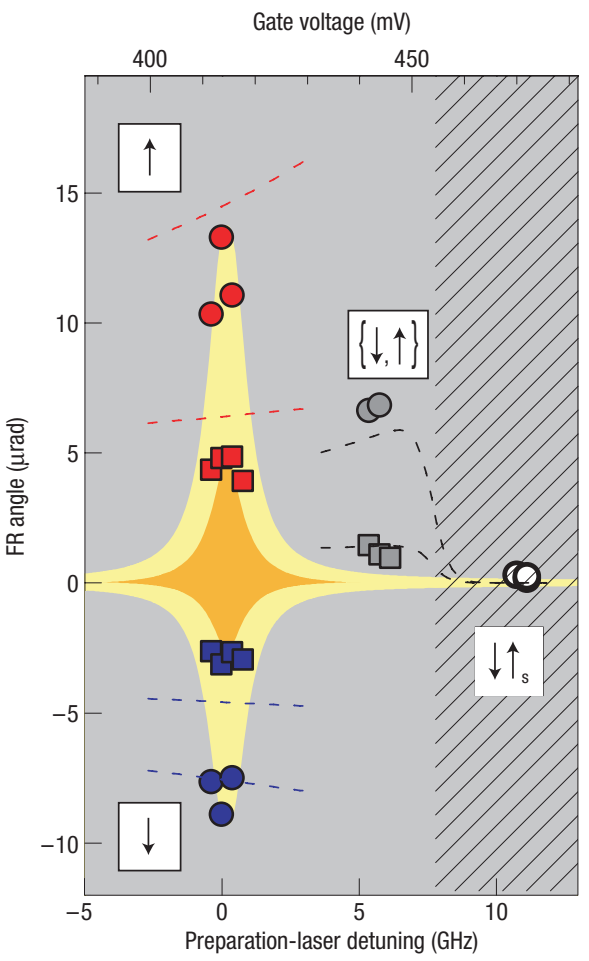

Figure 2 Spin-dependent FR signal from a QD. a, Top: The black circles correspond to the dispersive signal when the preparation laser is left far-detuned from either of the two Zeeman transitions, and has no effect on the electron spin, while the probe-laser detuning is varied from 30 to $45 \mathrm{GHz}$ with respect to the [ $\left.\sigma^{(+)}\right]$-polarized trion transition, with a laser power of $2 \mu \mathrm{W}$. The red circles correspond to the signal when the preparation laser is in the vicinity of the red Zeeman transition. At a gate voltage of $415 \mathrm{mV}$, the preparation laser hits resonance with the $\left[\sigma^{(-)}\right]$-polarized Zeeman transition and the electron is cooled to the spin-up state with near-unity fidelity. Bottom: Again at a gate voltage of $415 \mathrm{mV}$, the electron is cooled in the spin-down state by the preparation laser that is resonant with the $\left[\sigma^{(+)}\right]$-polarized Zeeman transition. The dispersive signal from a single electron spin is recovered, but with opposite sign, indicating a change of direction of FR. $\mathbf{b}$, The FR angle obtained for various probe- and preparation-laser detunings; the red (blue) circles correspond to the FR angle in response to the spin-up (spin-down) prepared state at $\sim 30 \mathrm{GHz}$ ( $56 \mathrm{GHz}$ ) detuning. The red (blue) squares correspond to the FR angle for a probe detuning of $66 \mathrm{GHz}(92 \mathrm{GHz}$ ). The corresponding grey circles (squares) indicate the FR angle when the electron spin is left in a thermal mixed state. The white circles indicate the signal level when the QD has a spin singlet of two electrons. The dashed curves indicate the theoretically expected FR angle for each case studied experimentally. The hatched areas mark the gate voltage values for which the quantum dot is charged with two electrons. The yellow and orange areas are to guide the eye.

We now consider probe-laser detunings that are larger than Zeeman splitting $\left(\Delta>E_{\text {Zeeman }}\right)$, where the difference signal arises from a competition between the two transitions. Figure 2a (top) shows the difference signal when the probe laser is blue-detuned by 20 to $40 \mathrm{GHz}$ with respect to the $\left[\sigma^{(+)}\right]$-polarized transition obtained in a $60 \mathrm{~s}$ measurement timescale per point. The black circles show the difference (offset) signal when the preparation laser is left detuned from the two Zeeman transitions: in this case, no state preparation is implemented and the electron spin state is effectively thermalized being close to a completely mixed state, as spin-flip events occur on timescales shorter than our measurement timescale. As the gate voltage is increased, the Zeeman-split optical transitions experience an equal strength of d.c.-Stark shift. Consequently, the detuning of the probe laser with respect to the two transitions is also decreased, creating an offset signal in accordance with the incommensurate detunings and partial cancellation of the FRs.

The red circles show the difference signal when the frequency of the weak preparation laser is in the near vicinity of the $\left[\sigma^{(-)}\right]$-polarized Zeeman transition. At a gate voltage of $415 \mathrm{mV}$, the preparation laser hits resonance and the electron is spin cooled to the spin-up state with near-unity fidelity due to state-mixinginduced spin-flip Raman transitions ${ }^{7}$. Therefore, we no longer observe the difference of two dispersive responses, rather the full signal due to one spin-state. Figure 2a (bottom) shows a similar measurement when the preparation laser is tuned to resonance with the $\left[\sigma^{(+)}\right]$-polarized Zeeman transition again at a gate voltage of $415 \mathrm{mV}$, preparing the electron in the spin-down state. The full dispersive signal from a single electron spin is recovered, but now with opposite sign, indicating FR in the opposite direction. The amplitude of this signal is less than that of Fig. 2a (top) in accordance with the additional detuning of $E_{\text {Zeeman }}=26 \mathrm{GHz}$ due to Zeeman splitting. Beyond a gate voltage of $450 \mathrm{mV}$, the QD charging state switches from one excess electron to two electrons, forming a spin-singlet ground state. As the trionic transitions are no longer present beyond this point, the laser experiences no dispersive response. Figure $2 \mathrm{~b}$ shows the $60 \mathrm{~s}$ time-averaged FR angle obtained from the dispersive signal for various probe-laser 

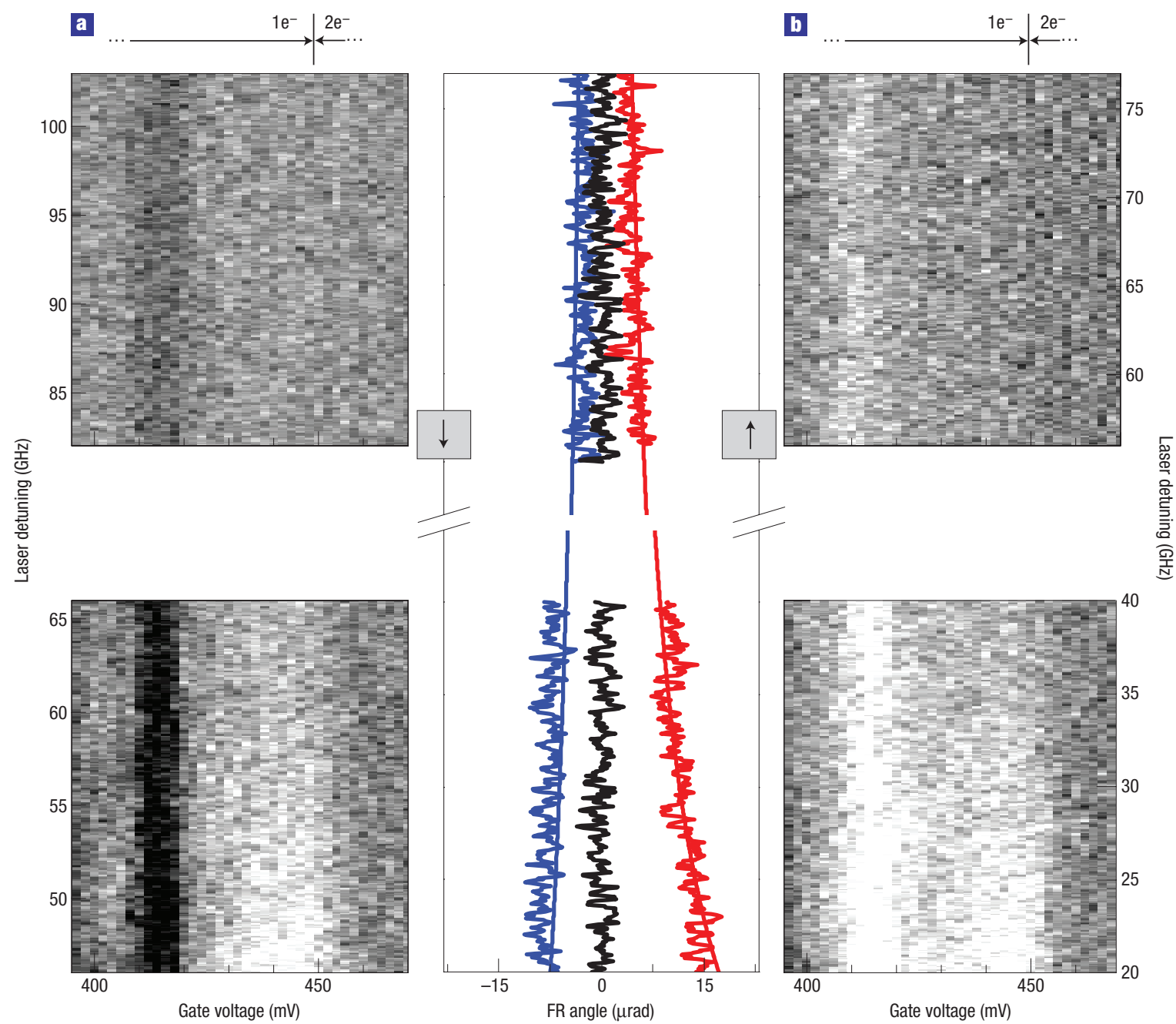

Figure 3 Full map of the dispersive signal as a function of gate voltage and probe-laser detuning. a, The preparation laser hits resonance at $415 \mathrm{mV}$, preparing the electron in the spin-down state. The signal here is obtained with a measurement timescale of $100 \mathrm{~ms}$ per data point. $\mathbf{b}$, Similar measurements when the electron is prepared in the spin-up state via optical pumping on the $\left[\sigma^{(-)}\right]$-polarized Zeeman transition. The dispersive signal strength at $415 \mathrm{mV}$ is shown in the middle plot as a cut along the blue (spin-down) and red (spin-up) curves along with the theoretically expected behaviour. The black curve in the middle plot is the signal at $470 \mathrm{mV}$ identifying the noise level.

and preparation-laser detunings. As expected, the FR angle reaches a positive (negative) maximum value when the pure spin-up (spindown) state is enforced. When the electron spin is left in a thermal mixed state, the value of the FR angle is reduced for probe-laser detunings ( $56 \mathrm{GHz}$ and $92 \mathrm{GHz}$ ) much larger than Zeeman splitting $(26 \mathrm{GHz})$. After submission of this work, we became aware of a similar time-averaged single-spin measurement, where partial spin preparation is achieved via an above-bandgap laser ${ }^{17}$.

Figure 3a shows a full map of the dispersive signal at a $100 \mathrm{~ms}$ measurement timescale per data point, plotted as a function of gate voltage and probe-laser detuning when the preparation laser is $\left[\sigma^{(+)}\right]$-polarized. The centre frequency of the probe laser is detuned $92 \mathrm{GHz}(\sim 200$ times $\gamma$ ) for the top figure and $56 \mathrm{GHz}$ for the bottom figure. Once again, a gate voltage of $415 \mathrm{mV}$ marks the preparation of the spin-down state. Figure $3 \mathrm{~b}$ shows a similar measurement when the preparation laser is $\left[\sigma^{(-)}\right]$-polarized to prepare the spin-up state on resonance. As a consequence of Zeeman splitting, the probe laser is detuned $66 \mathrm{GHz}$ for the top figure and $30 \mathrm{GHz}$ for the bottom figure from the nearest transition.
The signal from a spin in a mixed state remains identical, whereas the signal from the optically prepared spin switches sign. The middle plot shows line projections of the FR angle at $415 \mathrm{mV}$ for both cases, which are in accordance with the expected inverse detuning $\left(\Delta^{-1}\right)$ dependence.

Achieving spin read-out that is fast with respect to spinflip dynamics, that is, $T_{1}$ time, is only half the story. If, during this measurement, back-action on the measured observable $\left(\sigma_{z}\right)$ occurs on a timescale still faster than the natural spin-flip times, then the measured dynamics will be distorted by the back-action. Therefore, although the measurement time can be reduced with technical improvements, it is essential that the probability of a back-action event in the form of spin-flip Raman scattering remains small within the $T_{1}$ time, that is, $T_{\text {measure }}<T_{1}<T_{\text {back-action }}$. We cannot claim a quantum non-demolition or back-actionevading measurement here, as during the required measurement time to obtain a unity signal-to-noise level (100 ms), between 5 and 50 back-action events occur (for a branching ratio of $10^{-4}$ and $10^{-3}$, respectively). This limitation arises from the fact 
that our optical system has a numerical aperture of 0.5 and a photodetector efficiency of $10 \%$; under these conditions, $\left(\sigma_{\mathrm{QD}} / A_{\mathrm{L}}\right)$ cannot safely exceed the branching ratio. By using a combination of solid-immersion lenses matched to the refractive index of GaAs $\left(n_{\text {GaAs }}=3.5\right)$ and commercially available photodetectors with $80 \%$ efficiency, achieving a $\sim 100$-fold improvement of $\left(\sigma_{\mathrm{QD}} / A_{\mathrm{L}}\right)$ is plausible. It would then be possible to effectively eliminate spinflip Raman scattering from the probe laser within the anticipated measurement time of $\sim 10 \mathrm{~ms}$. In fact, on the basis of the predicted phonon $^{18,19}$ and cotunnelling ${ }^{20}$ limited spin-flip times, we could imagine resolving spin quantum jumps with these improvements. Alternatively, incorporating gated structures into photonic crystal nanocavities ${ }^{21}$ is demanding, but the existence of a far-detuned cavity mode could well be the way to obviate measurement back-action, at the same time allowing for coherent resonant manipulation of spins.

\section{METHODS}

\section{SAMPLE STRUCTURE AND APPARATUS}

The self-assembled InAs/GaAs QDs used in this experiment are grown by molecular beam epitaxy. Emission is blue-shifted by the partially covered island technique. The QDs are embedded in a Schottky-type heterostructure to achieve charge tuneability ${ }^{22}$. The sample composition from the bottom substrate to the top surface is as follows: GaAs substrate $\backslash 20 \mathrm{~nm} n-G a A s$ with $4 \times 10^{18} \mathrm{~cm}^{-3}$ doping level $\backslash 35 \mathrm{~nm}$ GaAs tunnel barrier $\backslash \mathrm{QD}$ layer $\backslash 12 \mathrm{~nm}$ $\mathrm{GaAs} \backslash 50 \mathrm{~nm} \mathrm{Al} \mathrm{Al}_{0.4} \mathrm{Ga}_{0.6} \mathrm{As} \backslash 88 \mathrm{~nm}$ GaAs $\backslash 5 \mathrm{~nm}$ titanium window. The bias voltage between the titanium window and the $n-G a A s$ layer determines the electric field across the structure and controls the charging of the QDs.

All measurements are carried out in a liquid helium bath cryostat operating at $4.2 \mathrm{~K}$. The numerical aperture of the confocal microscope system is 0.55 , resulting in a diffraction-limited laser spot with a diameter of $\sim 1 \mu \mathrm{m}$. The QD density of the sample is chosen to yield essentially one QD at a time within the focal area and its optical transitions can be identified by their spectral signatures. A magnetic field of up to $10 \mathrm{~T}$ in Faraday configuration can be applied using a superconducting magnet. Piezo-electric positioners are used to move the sample in all three dimensions.

\section{OPTICAL MEASUREMENT TECHNIQUES}

For the initial characterization and identification of the QD optical transitions and charging states, conventional micro-photoluminescence as a function of gate voltage is carried out ${ }^{23}$. The excitation laser energy is $1.6 \mathrm{eV}$ and the collected photoluminescence signal is sent to a liquid-nitrogen-cooled charge-coupled device $\left(\mathrm{LN}_{2}-\mathrm{CCD}\right)$ through a $0.75-\mathrm{m}$ spectrometer with a 1,500 lines $\mathrm{mm}^{-1}$ diffraction grating. The spectral resolution of this detection system is $\sim 30 \mu \mathrm{eV}$.

Once the two optical transitions of the single-electron-charged QD are identified, a polarization-sensitive differential transmission technique is used. The excitation laser is tuned to the vicinity of the optical frequency of the relevant transition, which in turn can be tuned by gate voltage, and the transmitted laser light on scattering from the QD is detected on the other side of the sample by a linear polarization analyser unit. This detection unit is composed of a polarizing beam splitter separating the linear basis X and Y polarization components of the light and redirecting each to a silicon-based $\mathrm{p}-\mathrm{i}-\mathrm{n}$ diode photodetector, as indicated in Fig. 1b. The spectral resolution of this technique is $\sim 0.08 \mu \mathrm{eV}(20 \mathrm{MHz})$ as dictated by gate-voltage noise and laser stability. Owing to the level of low-frequency noise of the system, lock-in detection is used following a $3.34 \mathrm{kHz}$ gate-voltage modulation ${ }^{24}$. The detection system is laser shot-noise limited for an off-resonant probe-laser power of $2 \mu \mathrm{W}$.

Received 13 October 2006; accepted 19 December 2006; published 28 January 2007.

\section{References}

1. DiVincenzo, D. P. The physical implementation of quantum computation. Fortsch. Phys. $\mathbf{4 8}$ 771-783 (2000).

2. Awschalom, D. D. \& Kikkawa, J. M. Electron spin and optical coherence in semiconductors. Phys. Today 52, 33-38 (June 1999).

3. Kato, Y. K., Myers, R. C., Gossard, A. C. \& Awschalom, D. D. Observation of the spin Hall effect in semiconductors. Science 306, 1910-1913 (2004).

4. Li, Y. Q. et al. Cavity enhanced Faraday rotation of semiconductor quantum dots. Appl. Phys. Lett. 88, 193126 (2006).

5. Elzerman, J. M. et al. Single-shot read-out of an individual electron spin in a quantum dot. Nature 430, 431-435 (2004).

6. Koppens, F. H. L. et al. Driven coherent oscillations of a single electron spin in a quantum dot. Nature 442, 766-771 (2006).

7. Atature, M. et al. Quantum-dot spin-state preparation with near-unity fidelity. Science 312, $551-553(2006)$

8. Ippolito, S. B., Goldberg, B. B. \& Ünlü, M. S. High spatial resolution subsurface microscopy. Appl. Phys. Lett. 78, 4071-4073 (2001).

9. Liu, Z. et al. High resolution, high collection efficiency in numerical aperture increasing lens microscopy of individual quantum dots. Appl. Phys. Lett. 87, 071905 (2005).

10. Dutt, M. V. G. et al. Stimulated and spontaneous optical generation of electron spin coherence in charged GaAs quantum dots. Phys. Rev. Lett. 94, 227403 (2005).

11. Grangier, P., Levenson, J. A. \& Poizat, J. P. Quantum non-demolition measurements in optics. Nature 396, 537-542 (1998).

12. Sugita, S., Machida, S. \& Yamamoto, Y. Quantum nondemolition measurement of a single electron spin in a quantum dot. Preprint at <http://www.arxiv.org/quant-ph/0301064> (2003).

13. Liu, R.-B., Yao, W. \& Sham, L. J. Coherent control of cavity quantum electrodynamics for quantum nondemolition measurements and ultrafast cooling. Phys. Rev. B 72, 081306 (2005).

14. Calarco, T., Datta, A., Fedichev, P., Pazy, E. \& Zoller, P. Spin-based all-optical quantum computation with quantum dots: Understanding and suppressing decoherence. Phys. Rev. A 68, 012310 (2003).

15. Petta, J. R. et al. Coherent manipulation of coupled electron spins in semiconductor quantum dots. Science 309, 2180-2184 (2005).

16. Alén, B. et al. Absorptive and dispersive optical responses of excitons in a single quantum dot. Appl. Phys. Lett. 89, 123124 (2006).

17. Berezovsky, J. et al. Nondestructive optical measurements of a single electron spin in a quantum dot. Science 314, 1916-1920 (2006).

18. Golovach, V. N., Khaetskii, A. \& Loss, D. Phonon-induced decay of the electron spin in quantum dots. Phys. Rev. Lett. 93, 016601 (2004).

19. Kroutvar, M. et al. Optically programmable electron spin memory using semiconductor quantum dots. Nature 432, 81-84 (2004).

20. Smith, J. M. et al. Voltage control of the spin dynamics of an exciton in a semiconductor quantum dot. Phys. Rev. Lett. 94, 197402 (2005).

21. Badolato, A. et al. Deterministic coupling of single quantum dots to single nanocavity modes. Science 308, 1158-1161 (2005).

22. Drexler, H., Leonard, D., Hansen, W., Kotthaus, J. P. \& Petroff, P. M. Spectroscopy of quantum levels in charge-tunable InGaAs quantum dots. Phys. Rev. Lett. 73, 2252-2255 (1994).

23. Warburton, R. J. et al. Optical emission from a charge-tunable quantum ring. Nature $\mathbf{4 0 5}$, 926-929 (2000).

24. Alén, B., Bickel, F., Karrai, K, Warburton, R. J. \& Petroff, P. M. Stark-shift modulation absorption spectroscopy of single quantum dots. Appl. Phys. Lett. 83, 2235-2237 (2003).

\section{Acknowledgements}

This work was supported by NCCR Quantum Photonics. The authors acknowledge many useful discussions with G. Giedke, A. Högele, C. Galland, G. Fernandez, J. Taylor, J. Grond, and G. Salis. M.A. and J.D. would like to thank J. Cash for technical assistance.

Correspondence and requests for materials should be addressed to M.A. and A.I.

Competing financial interests

The authors declare that they have no competing financial interests.

Reprints and permission information is available online at http://npg.nature.com/reprintsandpermissions/ 